\title{
Pumping of Nuclear Spins by Optical Excitation of Spin-Forbidden Transitions in a Quantum Dot
}

\author{
E. A. Chekhovich, ${ }^{1,2}$ M. N. Makhonin, ${ }^{1}$ K. V. Kavokin, ${ }^{1,3}$ A. B. Krysa, ${ }^{4}$ M. S. Skolnick, ${ }^{1}$ and A. I. Tartakovskii ${ }^{1}$ \\ ${ }^{1}$ Department of Physics and Astronomy, University of Sheffield, Sheffield S3 7RH, United Kingdom \\ ${ }^{2}$ Institute of Solid State Physics, 142432, Chernogolovka, Russia \\ ${ }^{3}$ A. F. Ioffe Physico-Technical Institute, 194021, St. Petersburg, Russia \\ ${ }^{4}$ Department of Electronic and Electrical Engineering, University of Sheffield, Sheffield S1 3JD, United Kingdom
}

(Received 29 June 2009; published 12 February 2010)

\begin{abstract}
We demonstrate that efficient optical pumping of nuclear spins in semiconductor quantum dots (QDs) can be achieved by resonant pumping of optically forbidden transitions. This process corresponds to oneto-one conversion of a photon absorbed by the dot into a polarized nuclear spin, and also has potential for initialization of hole spin in QDs. We find that by employing this spin-forbidden process, nuclear polarization of $65 \%$ can be achieved, markedly higher than from pumping the allowed transition, which saturates due to the low probability of electron-nuclear spin flip-flop.
\end{abstract}

Resonant optical pumping is a powerful method for direct control of individual quantum states in a variety of physical systems, including semiconductor quantum dots (QDs) [1-5]. In III-V semiconductor nanostructures the optically controlled carrier spin dynamics are strongly influenced by the hyperfine coupling of the electron spin with a bath of nuclei. The hyperfine interaction (HI) can be an advantage, for example enabling resonant optical pumping of the hole spin in charged dots [2,4], but it can also be a disadvantage limiting the electron spin coherence and lifetime [6] and therefore impeding electron spin manipulation in nanostructures [5,7]. Such undesirable effects arise from fluctuations of the effective nuclear magnetic field and can be suppressed by locking optical transitions in the dot to a resonant laser $[5,8]$, or by pumping nuclear spins into a suitable narrow distribution of states [7]. Another widely discussed approach is to pump very high nuclear spin polarizations [9], a task so far eluding an experimental realization.

Here we address the long standing problem of the realization of large nuclear polarization in semiconductors. Optical pumping is known to be an effective method for creating nuclear polarization [10-13]. However, full polarization of nuclei by this technique encounters two major difficulties: the low probability of electron-nuclear spin flip-flop due to the large electron Zeeman energy; and fast removal of the electron after its spin flip in order to allow a new pumping cycle to take place and avoid saturation of the pumping rate $[9,14]$. In this Letter we demonstrate an efficient nuclear spin pumping method which enables the above difficulties to be overcome by optical excitation of a spin-forbidden transition in a hole-charged quantum dot. The underlying second order process consists of absorption of a single photon accompanied by the simultaneous spin flip of an electron and a single nucleus. This mechanism, enabled by partial lifting of optical selection rules due to the transverse component of the nuclear magnetic field [15], is not limited by the energy splitting mismatch of the electron and nucleus. In both experiment and theory we find the counterintuitive result that this spin pumping method leads to higher degrees of nuclear polarization in the dot than those achieved by resonant and nonresonant pumping of nuclear spins via allowed optical transitions. We find that for the pumping via the spinforbidden process, the nuclear polarization increases with the laser intensity and saturates at $65 \%$, very close to the highest polarization degree reported for nonresonant excitation in QDs [10-13]. Using the results of powerdependent measurements in a wide range of external magnetic fields $B_{z}>1 \mathrm{~T}$ we show that this saturation is not due to the saturation of the rate of the pumping cycle. This indicates that similar polarization degrees at saturation observed in this and previous work may be due to inherent properties of the interacting electron-nuclear spin system, setting a fundamental limit to the maximum polarization achievable by optical pumping.

The experiments were performed on individual selfassembled InP/GaInP QDs [11] at a temperature of $4.2 \mathrm{~K}$, in external magnetic field $B_{z}$ up to $8 \mathrm{~T}$ perpendicular to the sample surface. We study positively charged QDs emitting at $\sim 1.84 \mathrm{eV}$. The sample was not intentionally doped. The dot charging with holes, verified by magnetospectroscopy and Hanle effect measurements, occurred due to residual doping.

The observation of nuclear spin pumping in this work has been realized using pump-probe techniques, where the effect of the resonant pump excitation is determined by monitoring the whole spectrum of the dot using a nonresonant probe, in contrast to the selective spectral probing used in absorption measurements $[1,2,4,5]$. The pumpprobe experiment cycle is shown schematically in Fig. 1(a). A long $(0.15 \mathrm{~s})$ circularly polarized pump pulse from a tunable single-mode laser excites the dot. A short $(0.5 \mathrm{~ms})$ probe pulse from a second laser, cross-circularly polarized relative to the pump and emitting below the GaInP band gap at $\sim 1.88 \mathrm{eV}$, is used to excite photo- 


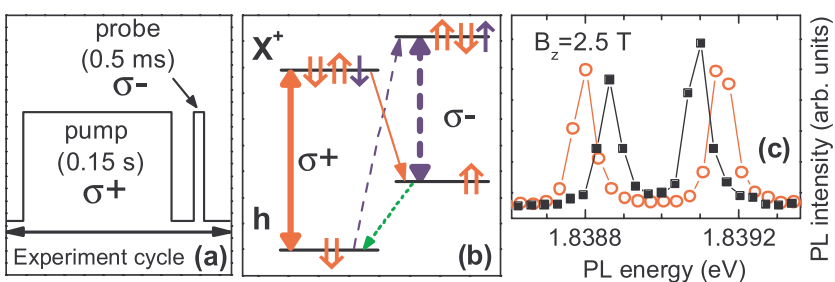

FIG. 1 (color online). (a) Pulse sequence used in the resonant nuclear spin pump-probe experiment. (b) Energy level diagram of a positively charged dot in magnetic field $B_{z}$. Electron and hole spin up (down) states are shown by $\uparrow(\downarrow)$ and $\Uparrow(\downarrow)$, respectively. Long thick (thin) arrows show "allowed" ("forbidden") optical transitions. Dotted arrow shows hole spin relaxation. (c) PL spectra of a positively charged dot measured with the probe pulse at $B_{z}=2.5 \mathrm{~T}$ for $B_{N} \approx 0$ (solid symbols) and at $B_{N} \approx-1.5 \mathrm{~T}$ (open symbols). Here the optics were optimized to maximize the PL signal, leading to differing sensitivity in detection of light with $\sigma^{+}$and $\sigma^{-}$polarizations and effectively unpolarized PL spectra.

luminescence (PL) from the positive trion $X^{+}$and to probe the states of the dot. This enables absolute $X^{+}$transition energies to be determined and effective nuclear (Overhauser) fields for a given pump laser wavelength to be deduced. Multiple measurement cycles were repeated for each resonant laser frequency to improve the signal-tonoise ratio, yielding a typical measurement time of $1 \mathrm{~min}$, exceeding the time required for the nuclear polarization to reach its steady-state value $\left(\tau_{\text {buildup }} \approx 10 \mathrm{~s}\right.$ ).

A diagram of the energy levels of the positively charged dot in external magnetic field $B_{z}$ is shown in Fig. 1(b). The system ground state with heavy hole spin up (down) $|\Uparrow\rangle(|\downarrow\rangle)$ and the photo-excited $X^{+}$trion state with electron spin up (down) $|\Uparrow \downarrow \downarrow \uparrow\rangle(\mid \Uparrow \downarrow \downarrow \downarrow)$ have energies $E_{\Uparrow(\Downarrow)}=$ $\pm \mu_{B} g_{h} B_{z} / 2$ and $E_{\Uparrow \Downarrow \uparrow(\Uparrow \Downarrow) ~}=E_{X^{+}} \pm \mu_{B} g_{e}\left(B_{z}+B_{N}\right) / 2$ respectively, where $\mu_{B}$ is the Bohr magneton, $B_{N}$ is the nuclear field, $g_{e(h)} \approx+1.5(+3.1)$ is the electron (hole) $g$ factor, and $E_{X^{+}}$is the PL energy at $B_{z}=B_{N}=0$. Allowed $\sigma^{-(+)}$polarized optical transitions from $\left.|\Uparrow \downarrow \downarrow\rangle\right\rangle(|\Uparrow \downarrow \downarrow\rangle)$ to $|\Uparrow\rangle(|\downarrow\rangle)$ observed in PL are shown by thick arrows.

The magnitude of the nuclear field $B_{N}$ is deduced from

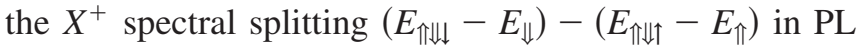
excited by the probe. Typical PL spectra at two different $B_{N}$ are shown in Fig. 1(c). The effect of the resonant excitation with a $\sigma^{+}$polarized pump laser is demonstrated in Fig. 2, where $B_{N}$ is shown as a function of the pump laser energy $E_{l}$.

As shown in Fig. 2(a) for the case of high magnetic field $B_{z}=2.5 \mathrm{~T}$ and laser power $P_{\text {res }}=15 \mu \mathrm{W}$, the dependence of $B_{N}$ on laser frequency has the form of two strongly asymmetric dips. When the laser is tuned from low energy towards resonance with the optically allowed transition $|\Downarrow\rangle \leftrightarrow|\Uparrow \downarrow \downarrow\rangle, B_{N}$ decreases from $0 \mathrm{~T}$ to $-0.6 \mathrm{~T}$ and then switches abruptly to $B_{N} \sim-0.2 \mathrm{~T}$ with a further slow increase to $\sim 0 \mathrm{~T}$. Surprisingly, another strong decrease of $B_{N}$ is observed when the laser is tuned near the

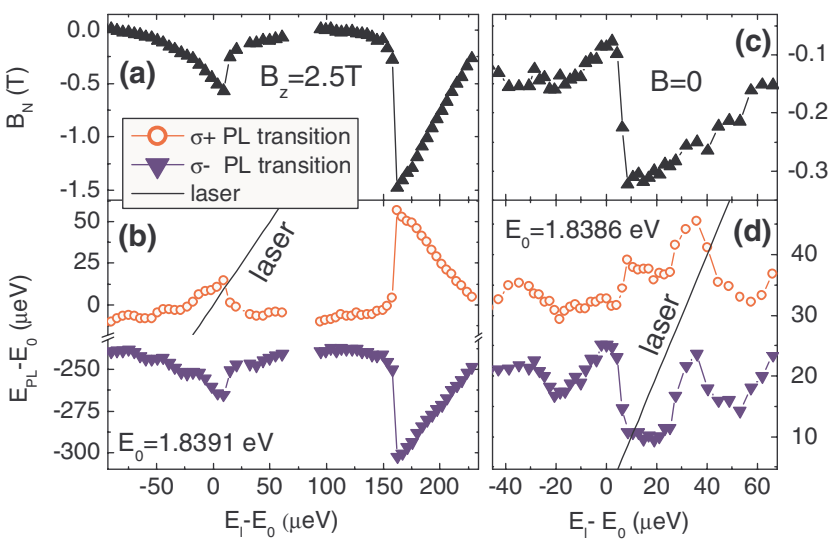

FIG. 2 (color online). Overhauser field $B_{N}$ on the dot (a),(c) and PL transitions energies $E_{\mathrm{PL}}$ (b),(d) as a function of the energy $E_{l}$ of the $\sigma^{+}$polarized resonant laser with excitation power $P_{\text {res }}=15 \mu \mathrm{W}$ at $B_{z}=2.5 \mathrm{~T}$ (a),(b) and $B_{z}=0$ (c),(d). The full lines on panels (b) and (d) show the laser energy.

spin-forbidden transition $|\Downarrow\rangle \leftrightarrow|\Uparrow \downarrow \downarrow \uparrow\rangle$ [thin dashed arrow in Fig. 1(b)]. Here in marked contrast, an abrupt change in $B_{N}$ from 0 to $-1.5 \mathrm{~T}$ occurs at low laser energy, with the maximum $\left|B_{N}\right|$ at this optical power 2.5 times larger than that excited via the spin-allowed transition. When $E_{l}$ is tuned further, $B_{N}$ gradually increases back to 0 .

The results in Fig. 2(a) are deduced from the measurement of absolute $X^{+}$PL transitions energies shown in Fig. 2(b) as a function of laser energy. The detuning between the $\sigma^{+}$PL line and the laser at each $E_{l}$ is given by the energy difference between the circles and the black line in Fig. 2(b), which represents the energy of the laser. In the case of the spin-allowed process the energy of the optical transition shifts with the tuning of the laser, similar to the "dragging" effect reported in Ref. [16]. The abrupt change of nuclear polarization occurs just as the laser is tuned slightly above the $\sigma^{+}$PL line.

The same laser tuning experiment was repeated at $B=0$ [see Figs. 2(c) and 2(d)]. Here, an abrupt change in $B_{N}$ occurs only when the $\sigma^{+}$polarized laser comes into resonance with the $\sigma^{-}$polarized $|\Uparrow\rangle \leftrightarrow|\Uparrow \downarrow \downarrow\rangle$ PL transition coinciding with the forbidden $|\downarrow\rangle \leftrightarrow|\Uparrow| \downarrow\rangle\rangle$ transition at $B=0$. Also, the asymmetry of the resonance curve is similar to that observed for pumping via the spin-forbidden transition at $B_{z}=2.5 \mathrm{~T}$ in Fig. 2(a): the abrupt change in $B_{N}$ is observed towards lower laser energy.

In order to explain the observed effects we consider the resonant nuclear spin pumping mechanism illustrated in Fig. 1(b). As the QD contains of the order of $10^{4}$ magnetic nuclei, their polarization requires many cycles of optical excitation or recombination. The optically allowed circularly polarized transitions shown in Fig. 1(b) with thick arrows conserve spin and cannot be responsible for nuclear spin pumping. Because of the mismatch of the electron and nuclear Zeeman energy, nuclear spin pumping occurs only in a second order process consisting of emission or absorption of a photon accompanied by simultaneous flip-flop of 
electron and nuclear spins. These transitions [thin lines in Fig. 1(b)] are optically forbidden and become weakly allowed due to mixing of the two $X^{+}$states by the hyperfine interaction. The magnitude of wave function mixing $\alpha=\mu_{B} g_{e}\left\langle\delta B_{N, \perp}^{2}\right\rangle^{1 / 2} / E_{e Z}$ is governed by the meansquared in-plane fluctuation of the Overhauser nuclear field $\left\langle\delta B_{N, \perp}^{2}\right\rangle^{1 / 2}$ and by the electron Zeeman splitting of

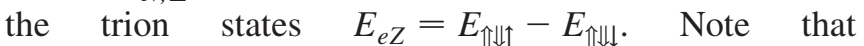
$\mu_{B} g_{e}\left\langle\delta B_{N, \perp}^{2}\right\rangle^{1 / 2} \approx 1 \mu \mathrm{eV}$, and therefore in our experiments $\alpha$ is always small.

For the $\sigma^{+}$polarized excitation used in Fig. 2, there are two possible cyclic processes yielding nuclear polarization. Both start from the spin-down state of the resident hole $|\downarrow\rangle$ and involve optically forbidden transitions: (1) The cycle associated with the spin-allowed (SA) process consists of the following steps: (i) excitation of the allowed transition to the spin-down $|\Uparrow \backslash \downarrow\rangle\rangle$ state [thick solid arrow in Fig. 1(b)]; (ii) recombination to the spin-up hole state $|\Uparrow\rangle$ (thin solid arrow), assisted by HI; (iii) hole spin flip back into the $|\Downarrow\rangle$ state (dotted arrow). (2) The cycle associated with the optically spin-forbidden (SF) process also involves three steps: (i) HI-assisted excitation of the spin-up $|\Uparrow \downarrow \downarrow \uparrow\rangle$ state (thin dashed arrow); (ii) $X^{+}$recombination via the allowed transition into the $|\Uparrow\rangle$ state (thick dashed arrow); (iii) and, finally, the hole spin flip completing the cycle.

Each of the above excitation cycles results in a change of the $Z$ projection of the total nuclear spin in the QD by -1 . We calculate the total rate of spin pumping into the nuclear system $W_{\text {pump }}$ as the sum of the rates of these cycles. We make a simplifying assumption that the rate of hole spin relaxation $1 / \tau_{h}$ is fast compared to the rate of the electronnuclear spin flip-flop, which allows us to calculate $W_{\text {pump }}$ by solving optical Bloch equations for the two photoexcitation paths. We return to this assumption below.

For the SF cycle, the lifetime $\tau$ of the excited state is determined by the allowed recombination transition. In contrast, for the SA cycle the recombination rate into the spin-up hole state is reduced by the factor $\alpha^{2}$. The hyperfine admixture factor $\alpha$ also governs the optical matrix element for the excitation transition in the SF cycle, which is given by $\alpha \Omega_{R}$ rather then $\Omega_{R}$ as in the case of the SA cycle (here $\Omega_{R} \sim \sqrt{P_{\text {res }}}$ is the laser Rabi frequency). As a result, we obtain the following expressions for the rates of SA and SF cycles:

$$
\begin{aligned}
& W_{\mathrm{SA}}=-\frac{\alpha^{2} \tau^{-1}\left(\tau^{*} \Omega_{R}\right)^{2} / 2}{1+\left(\tau^{*} \Omega_{R}\right)^{2}+\left(E_{\text {介॥ }}-E_{\Downarrow}-E_{l}\right)^{2} \tau^{* 2} \hbar^{-2}} \\
& W_{\mathrm{SF}}=-\frac{\tau^{-1}\left(\alpha \tau^{*} \Omega_{R}\right)^{2} / 2}{1+\left(\alpha \tau^{*} \Omega_{R}\right)^{2}+\left(E_{\text {介 } \downarrow}-E_{\Downarrow}-E_{l}\right)^{2} \tau^{* 2} \hbar^{-2}},
\end{aligned}
$$

where $\tau^{*}<\tau$ describes trion spin state broadening by radiative damping and other dephasing processes. At low pumping $\left[\left(\tau^{*} \Omega_{R}\right)^{2} \ll 1\right]$ both processes result in identical resonance line shapes, shifted in energy by the splitting of the $X^{+}$states. However, with increasing pump intensity,
$W_{\mathrm{SA}}$ saturates at $\alpha^{2} \tau^{-1} / 2$ for $\left(\tau^{*} \Omega_{R}\right)^{2} \approx 1$, while $W_{\mathrm{SF}}$ grows linearly with pump intensity up to $\left(\tau^{*} \Omega_{R}\right)^{2} \approx$ $\alpha^{-2} \gg 1$ where it saturates at $\tau^{-1} / 2$.

The stationary value of the nuclear field is found from the condition that the total pumping rate $W_{\text {pump }}$ via SF and SA processes must equal the loss rate of nuclear polarization:

$$
W_{\mathrm{SA}}\left(B_{N}, E_{l}\right)+W_{\mathrm{SF}}\left(B_{N}, E_{l}\right)=\gamma N \frac{B_{N}}{B_{N, \max }},
$$

where $\gamma$ is the rate of nuclear spin loss via spin relaxation and diffusion, $N$ is the number of nuclei in the $\mathrm{QD}, B_{N \text {,max }}$ is the maximum $\left|B_{N}\right|$ corresponding to $100 \%$ polarized nuclear spins $\left(\mu g_{e} B_{N \text {,max }}=230 \mu \mathrm{eV}\right.$ in InP [17]).

Numerical solutions of the nonlinear equation Eq. (2) for values of parameters relevant to our experiment are shown in Fig. 3. The Overhauser field $B_{N}$ (dots) and nuclear spin pumping rate $\left|W_{\text {pump }}\right|$ at $B_{N}=0$ are shown in panels (a) and (c) for $B_{z}=2.5 \mathrm{~T}$ and $B=0$ respectively. Calculated PL transition energies $E_{\Uparrow \Perp \downarrow}-E_{\Downarrow}$ and $E_{\Uparrow \Downarrow \downarrow}-E_{\Uparrow}$ are shown in panels (b) and (d) with symbols, and the pump laser energy is shown by the continuous lines.

The behavior calculated with realistic parameters is in excellent agreement with the experimental data in Fig. 2. We used nuclear polarization fluctuation $\mu_{B} g_{e}\left\langle\delta B_{N, \perp}^{2}\right\rangle^{1 / 2} \approx 1.53(1.05) \mu \mathrm{eV}$, electron spin level broadening $\hbar / \tau^{*} \approx 6.8(5.5) \mu \mathrm{eV}$ and excitation power $\Omega_{R} \tau^{*} \approx 2.0(3.5)$ at $B_{z}=0(2.5) \mathrm{T}$. As expected, the best fits reflect the strong suppression of the nuclear spin depolarization rate in high magnetic fields: the product of $\tau \gamma N \approx 5 \times 10^{-2}$ at $B=0$ and decreases down to $\approx 8 \times 10^{-5}$ at $B_{z}=2.5 \mathrm{~T}$.

The origin of the asymmetric shapes of the resonances in Figs. 2 and 3 lies in the nonlinear dependence on $B_{N}$ of both the trion state detuning from the laser and the nuclear


FIG. 3 (color online). Results of calculations using Eqs. (1) and (2) for $B_{z}=2.5 \mathrm{~T}$ (a),(b) and $B=0$ (c),(d): calculated nuclear field $B_{N}$ on the dot (a),(c) and PL transitions energies $E_{\mathrm{PL}}(\mathrm{b}),(\mathrm{d})$ as functions of the energy $E_{l}$ of the $\sigma^{+}$polarized laser [(crosses) circles show (meta) stable solutions]. Lines on panels (b)and (d) show the laser energy. Lines in (a) and (c) show nuclear spin pumping rate $\left|W_{\text {pump }}\right|=\left|W_{\mathrm{SA}}+W_{\mathrm{SF}}\right|$ as a function of $E_{l}$ for a fixed $B_{N}=0$. 
spin pumping rate $W_{\text {pump }}$. The feedback arising when the laser approaches the spin-allowed $|\Downarrow\rangle \rightarrow|\Uparrow| \downarrow\rangle$ and spinforbidden $|\Downarrow\rangle \rightarrow|\Uparrow \downarrow \uparrow\rangle$ transitions has different signs, since with the buildup of negative $B_{N}$, the $|\Uparrow \downarrow \downarrow\rangle(|\Uparrow \Uparrow \downarrow \uparrow\rangle)$ state shifts to higher (lower) energy. Thus the asymmetric shape of the SA (SF) resonances exhibit the sharp drop in $\left|B_{N}\right|$ in the direction of high (low) $E_{l}$.

Calculations for the case of $B=0$ [Fig. 3(c) and 3(d)] also show an asymmetric resonance with the abrupt change in nuclear polarization on the low energy side, in agreement with the results in Fig. 2. As in experiment, the abrupt increase of $\left|B_{N}\right|$ under $\sigma^{+}$excitation is observed when the laser is in exact resonance with the $\sigma^{-}$PL line (transition to $|\Uparrow \downarrow \downarrow \uparrow\rangle$ state), demonstrating efficient nuclear spin pumping through the forbidden optical transition.

By performing power-dependent measurements in high magnetic field $\left(B_{z}>1 \mathrm{~T}\right)$, we find that the maximum nuclear polarization pumped via the spin-forbidden transition increases with power and saturates at $65 \%, 1.5$ and 1.2 times higher than the saturation levels observed for the SA cycle (45\%) and nonresonant excitation (55\%), respectively. In order to explain the saturation of nuclear polarization at a level considerably lower than $100 \%$ we first rule out the limiting influence of hole spin relaxation, a required step in both SF and SA cycles. Slowing of the nuclear spin pumping rate can take place in principle once $W_{\mathrm{SA}}$ or $W_{\mathrm{SF}}$ exceed $\tau_{h}^{-1}$, which may result in reduction of the maximum pumping rate in the SF process down to $W_{\mathrm{SF}}^{\max } \approx \tau_{h}^{-1}$. However, despite the strong enhancement of the hole spin relaxation rate with $B_{z}$ [18], we find that the nuclear polarization degree in the SF process saturates at the same level of $65 \%$ in a wide range of $B_{z}=1-8 \mathrm{~T}$. This indicates that the hole spin relaxation rate is considerably higher than the nuclear spin pumping rates achieved in the experiment [as was assumed in Eq. (1)].

According to Eq. (1), $W_{\mathrm{SA}}$ saturates at $\left(\tau^{*} \Omega_{R}\right)^{2} \approx 1$ due to saturation of the optically allowed transition, whereas $W_{\mathrm{SF}}$ is expected to grow linearly with laser intensity up to much higher pumping power $\left(\tau^{*} \Omega_{R}\right)^{2} \approx \alpha^{-2}, \alpha^{-2}>3000$ in a wide range of magnetic fields [19]. However, in the experiment $\left|B_{N}\right|$ in the $\mathrm{SF}$ process saturates at smaller power compared to the SA case.

We thus conclude that the saturation of nuclear polarization at $65 \%$ is not due to saturation of the forbidden transition itself or due to slow hole spin dynamics. These results of the power dependence (to be reported in detail elsewhere) suggest that the complete physical picture of the observed phenomena should also include additional mechanisms such as, for example, formation of dark nuclear states [9] or suppression of nuclear spin fluctuations at high optical pumping of the dot. The maximum degree of nuclear spin polarization achieved by optical pumping in different types of QDs is of a very similar magnitude [10-13], indicating common fundamental processes (yet to be fully understood), limiting the efficiency of the optical orientation of nuclear spins.
In conclusion, we have used techniques combining resonant pump with nonresonant probe to demonstrate nuclear spin pumping based on optically forbidden transitions in a positively charged quantum dot. This process first proposed in Refs. [15,20] is a close analogue of the "solid effect" [21], a dynamic nuclear polarization phenomenon observed in solids with paramagnetic centers under microwave excitation of dipole-forbidden transitions weakly allowed by the hyperfine interaction. The optical solid effect revealed in this work results in much higher degrees of nuclear polarization compared with resonant studies on negatively charged dots $[5,16]$, where only allowed transitions were employed. Another factor limiting the magnitude of nuclear polarization in previous studies may be interaction of the dots with an $n$-type contact, which may also lead to suppression of the "dragging" effect at low magnetic fields. In contrast, in our case the spin pumping is very efficient in a wide range of magnetic fields including $B_{z}=0$. Furthermore, both theory and experiment indicate that in realistic conditions the excitation of the forbidden transition is the most efficient mechanism of dynamic nuclear polarization.

We are grateful to A.J. Ramsay, V.I. Fal'ko, V.D. Kulakovskii, and V.L. Korenev for fruitful discussions. This work has been supported by the EPSRC Grants No. EP/G601642/1, No. EP/C54563X/1, the EPSRC IRC for QIP, and the Royal Society.

[1] M. Atature et al., Science 312, 551 (2006).

[2] B. D. Gerardot et al., Nature (London) 451, 441 (2008).

[3] A. J. Ramsay et al., Phys. Rev. Lett. 100, 197401 (2008).

[4] D. Brunner et al., Science 325, 70 (2009).

[5] X. Xu et al., Nature (London) 459, 1105 (2009).

[6] V. K. Kalevich, K. V. Kavokin, and I. A. Merkulov, Spin Physics in Semiconductors (Springer, New York, 2008).

[7] D. J. Reilly et al., Science 321, 817 (2008).

[8] A. Greilich et al., Science 313, 341 (2006).

[9] A. Imamoglu, E. Knill, L. Tian, and P. Zoller, Phys. Rev. Lett. 91, 017402 (2003)

[10] A. I. Tartakovskii et al., Phys. Rev. Lett. 98, 026806 (2007).

[11] J. Skiba-Szymanska et al., Phys. Rev. B 77, 165338 (2008).

[12] B. Urbaszek et al., Phys. Rev. B 76, 201301(R) (2007).

[13] D. Gammon et al., Phys. Rev. Lett. 86, 5176 (2001).

[14] M. Makhonin et al., Appl. Phys. Lett. 93, 073113 (2008).

[15] V. L. Korenev, Phys. Rev. Lett. 99, 256405 (2007).

[16] C. Latta et al., Nature Phys. 5, 758 (2009).

[17] B. Gotschy et al., Solid State Commun. 71, 629 (1989).

[18] D. Heiss et al., Phys. Rev. B 76, 241306(R) (2007).

[19] The forbidden transition was not observed in PL measurements, which taking into account the signal-to-noise ratio in PL provides the upper estimate for $\alpha^{2}<1 / 3000$.

[20] A. S. Bracker, D. Gammon, and V. L. Korenev, Semicond. Sci. Technol. 23, 114004 (2008).

[21] A. Abragam, The Principles of Nuclear Magnetism (Oxford University Press, London, 1961). 